\title{
The Radioactivity of Nuclei \& Solar Oscillations: New Experiments
}

\author{
Oleg Borisovich Khavroshkin, Vladislav Vladimirovich Tsyplakov \\ Schmidt Institute of Physics of the Earth, RAS, Moscow, Russia \\ Email:khavole@mail.ru
}

Received 7 December 2015; accepted 26 January 2016; published 29 January 2016

Copyright (C) 2016 by authors and Scientific Research Publishing Inc.

This work is licensed under the Creative Commons Attribution International License (CC BY). http://creativecommons.org/licenses/by/4.0/

c) (i) Open Access

\section{Abstract}

The experimental detection of the hidden periodicities in the activity of various radioactive sources which were observed by different instruments and which coincided with the period of the free oscillations of the Sun gave an impetus to the further research. The simultaneous recording of gamma rays from two radioactive sources revealed the elements of synchronism and the periods of solar oscillations as well as the phase delay for the different sources in the obtained time series. A neutrino detector has been designed and tested, the advanced schemes for neutrino detection are developed, and the impact of the neutrino source on the radioactive matter is explored. The search for the new principles for creating the emitters of neutrino beams is conducted.

\section{Keywords}

Radioactive Source, Periodicities of Radioactivity, Solar Oscillations and Neutrino, Abnormal Capture Cross Section, Neutrino Beams, Neutrino Detector

\section{Introduction}

This work continues one of the new trends in the experimental nuclear physics - the study of natural radioactivity as an object which is capable of changing its time characteristics and the informativity of this process [1] [2] which yielded the fundamentally new information.

1) The spectrum of the time variations in the activity of the sample from Cisbaikalian radioactive ore and other radioactive elements contains the peaks coinciding with the periods of the free oscillations of the Sun [2]-[5].

2) The capture cross section of the heavy deformed radioactive nucleus before the decay increases by many orders of magnitude and can interact with the flow of the solar neutrino modulated by the free oscillations of the Sun.

3) The spectrum of the long-period oscillations of the Earth, exceeding the free oscillations, contains the peaks 
which coincide within an accuracy of $1 \%, \ldots, 3 \%$ with the peaks of the free oscillations of the Sun. The mechanism of excitation of these oscillations corresponds to the nature of the activity variations in the radioactive ore sample.

4) These effects are part of the mechanisms of interaction in the Earth-Sun system. In particular, they affect the seismicity.

The analysis of the experimental works, both Russian and foreign, indicates that the most reliable results were obtained in [1]-[5], since [6]-[19] do not even provide a working hypothesis about the role of neutrinos, solar neutrinos and solar oscillations. Therefore, the studies [1]-[5] have absolute novelty. However, it should be noted that for accepting these data as indisputable, similar experiments carried out with the different instruments by a different procedure in a different laboratory are required. To this end, the study on hidden periodicities in the radioactive components of $\gamma$-radiation of barium 133, radium 226, americium 241 and cesium-137 [20] was carried out. This study in the most reliable way confirms all the previous results obtained in this new research direction in [1]-[5]. This enables the generalizations to be made based on both the cited works and on the studies in the other thematically close research fields, particularly in geophysics. So, the basic experimental result is that the spectrum of time variations in the activity of the sample of Cisbaikalian radioactive ore contains about 50 significant peaks coinciding with the periods of free oscillations of the Sun, and, as a most reliable consequence, it confirms the neutrino model of the effect [2] [3]. Specifically, the capture cross section of a heavy deformed radioactive nuclei at the pre-decay time instant increases by many orders of magnitude and can interact with the flow of solar neutrino modulated by the free oscillations of the Sun. This model logically continues the entire class of the detectors which make use of the metastable state of the working (recording) material (the Wilson cloud chamber, bubble chamber, and spark chamber) and, by its high potential, is incomparable with the radiochemical and Cherenkov detectors. Currently, the obtained results make it quite real to create a neutrino telescope with high resolution which is able to solve astrophysical problems and explore the Sun and the Earth's core. For example, many nuclear reactions occurring in the astrophysical objects are accompanied by neutrino emission. With a small section of neutrino absorption by the material $\left(\sigma=10-44 \mathrm{~cm}^{2}\right)$, the neutrino fluxes are barely absorbed by the Sun and other stars. The flow from the Sun makes up 2\% of the total energy; however, in the case of supernova outburst, it is by many orders of magnitude higher.

The spectrum of the long-period oscillations of the Earth exceeding the periods of the free oscillations of the Earth contains the peaks (more than 15) which, within an accuracy of $1 \%-3 \%$, coincide with the peaks of the free oscillations of the Sun. The excitation mechanism of these oscillations is similar to that driving the emergence of the variations in the activity of the radioactive ore sample [2] [3]. The revealed effects are part of the mechanisms of solar-terrestrial interaction; they affect seismic activity and enable one to search for a natural nuclear reactor in the inner core of the Earth.

Regardless of the in situ physical conditions of the heavy unstable radioactive nuclei, their decay process is modulated by the varying flow of solar neutrinos.

The activity level of heavy unstable radioactive nuclei as an open system is influenced by three parameters: the spin impact (spin speed), dynamic gravity (centrifugal force), and solar neutrino flux. Under certain relationships between these parameters, the elements of self-organization appear in the structure of the activity.

Some of these parameters of the radioactive source can be determined by the experimental.

According to Shestopalov, there is also a correlation between the neutrino fluxes and seismicity of the Earth. Shestopalov also hypothesized the possibility of neutrino formation during the periods of the strong deep earthquakes. To date, the studies covered the relationship between solar activity, Earth's seismic activity, and neutrino fluxes registered in the chlorine-argon experiment during 1970-1994. These studies revealed a negative correlation between the solar activity and seismicity of the Earth and a positive correlation between the seismic activity of the Earth and variations in the neutrino flux. It is hypothesized that the neutrinos which were registered in the chlorine argon experiment do not exclusively have a solar origin but are partly terrestrial. The qualitative mechanism of neutrino formation during the strong deep earthquakes is suggested [21]. The studies have been carried out on exploring the existence of the large masses of uranium in the central zones and the cores of planets including the Earth as well as on the probable modes and the nuclear chain reactions in the Earth's core [22]-[24]. The theoretical calculations and model experiments suggest that at the early stages of evolution of the Earth and other planets, the uranium and thorium oxides (or carbides), which are denser, high-melting, and marginally soluble, could settle down from the magma "ocean" onto the solid inner core of the planet. Billions years ago, the concentration of the fissile isotope $235 \mathrm{U}$ in natural uranium was sufficient to initiate the chain reactions 
in the developing active layer. In the case of low power, the self-sustaining chain reaction which began far in the past could be occasionally resumed up to the present time. A local or global explosion initiated by an asteroid impact could have occurred. The nuclear chain reactions could play and probably play important role in the evolution of the Earth and solar system overall [24]. We recapitulate the works [22]-[24] in such a detail because the results presented in the present paper (the strong interaction between solar neutrinos and radioactive elements in the laboratory conditions and in situ in the Earth) significantly strengthens the conclusions of the authors [22]-[24] whose scientific philosophy is used by the foreign scientists as a basis for constructing a new model of the formation of the Moon. The supernova explosions should now also be treated in a different way: about 40 years ago, the Czech scientists established a strong correlation between the outbursts and catastrophic earthquakes. The hypothesis of the Phaeton becomes now quite workable: if a planet had the geological structures with an extensive component of radioactive elements, the supernova explosion in the vicinity of the solar system, followed by a powerful stream of neutrinos, has led to the explosion of the planet. This is indirectly supported by the asteroids' surfaces which have huge craters formed by the impacts of the other celestial bodies whereas in accordance with the simplest geomechanical relations these asteroids should be fragmented. The preservation of the asteroids can only be due to the fact that their craters appeared as early as at the time when they were still part of the planet. This is confirmed by another fact: at the antipodal points, the craters do not have the signs of spall phenomena whereas their existence is necessary according to the theory and practice of impact mechanics.

Thus, the interaction between neutrinos and radioactive elements is of interest for the researchers from both the theoretical and applied standpoints, and this research is continued in a series of the experiments.

\section{Estimating (Testing) the Sensitivity of the Neutrino Sensor (NS)}

A NS sensitivity test was carried out with the use of Cs137 isotope. Cesium-137 is a daughter product of $\beta$-decay of 137Xe nuclide (the half-life is 3.818 (13) [2] min): Cesium-137 undergoes beta decay 137 Cs55-137Ba56 + $\mathrm{e}^{-}+$ve (the half-life is 30.17 years) which produces a stable Barium isotope $137 \mathrm{Ba}$. In 94.4 [4]\%, the decay occurs with intermediate formation of $137 \mathrm{Ba}$ - the nuclear isomer of barium-137 with a half-life of 2.55 min, which, in turn, passes into the ground state with the emission of gamma quantum with the energy of $661.7 \mathrm{keV}$ (or a conversion electron with the energy of $661.7 \mathrm{keV}$ minus the electron-binding energy). The total energy released by the beta decay of one cesium-137 nucleus is $1175.63 \pm 0.17 \mathrm{keV}$. Thus, cesium is not only a source of beta radiation but also of neutrinos whose total energy is $514 \mathrm{keV}$ and which form a continuous spectrum.

For testing the NS sensitivity, we used a specimen of Cs137 isotope with a mass of $\sim 3 \mathrm{~g}$. The specimen was placed in a lead container with a thickness $>1 \mathrm{~cm}$ in order to eliminate practically direct effects of all radiation types other than neutrinos. Then we counted the number of ND responses at a distance $\mathrm{L}=0.1,0.2,0.3,0.5$ and $1 \mathrm{~m}$ from the Cs137 neutrino source during $23 \mathrm{~s}$. In order to increase the reliability of the results, 25 measurements were conducted at each distance from the source and their average $\bar{X}$ and standard deviation $\sigma$ were calculated. The results of testing are presented in Table 1.

We note that starting from a distance of $0.3 \mathrm{~m}$, we obtain the background level, i.e. the NS only detects neutrinos at the distances of $0.1 \mathrm{~m}$ and, probably, $0.2 \mathrm{~m}$. Then, by interpolating the obtained values, we may assume that for the neutrino sources with a mass of 3 tons $=3 \times 10^{6} \mathrm{~g}$, distance $\mathrm{L}$ is $\sim 103 \mathrm{~m}=1 \mathrm{~km}$. These sources of neutrinos (or similar) may be All the beta emitting sources such as the operating nuclear reactors, radioactive waste repositories, uranium ore deposits, etc. can act as the neutrino sources of this (or similar) type.

\section{New Principles of Neutrino Detection}

In 1955, R. Davis set up an experiment to detect neutrinos from a nuclear reactor. He used the method suggested in 1946 by B. Pontecorvo. The idea of this method is as follows. The 37Ar nuclei experience e-capture, i.e. one of the protons forming the 37Ar nucleus is transformed into a neutron.

Table 1. Function $\sigma$ from distance.

\begin{tabular}{cccccc}
\hline$L(\mathrm{M})$ & 0.1 & 0.2 & 0.3 & 0.5 & 1 \\
$\bar{X}$ & 47.25 & 43.2 & 40.24 & 40.2 & 39.1 \\
$\sigma$ & 5.94 & 6.95 & 6.1 & 5.58 & 7.2 \\
\hline
\end{tabular}




$$
\mathrm{e}^{-}+37 \mathrm{Ar} \rightarrow v+37 \mathrm{Cl}
$$

The reverse reaction

$$
v+37 \mathrm{Cl} \rightarrow \mathrm{e}^{-}+37 \mathrm{Ar}
$$

should have the same cross section. By measuring the amount of the formed radioactive nuclei $37 \mathrm{Ar}(T 1 / 2=$ 35.04 days), we can estimate the neutrino fluxes. The theory predicts the interaction cross section $\sigma_{\text {theor }} \sim 10^{-43}$ $\mathrm{cm}^{2}$, whereas the experiment demonstrates a different result $\sigma_{\text {exp }}<0.25 \times 10^{-44} \mathrm{~cm}^{2}$. The neutrino detection in the gallium-germanium method is based on the reaction of neutrino interactions with gallium which produces germanium:

$$
71 \mathrm{Ga}+v \mathrm{e} \rightarrow 71 \mathrm{Ge}+\mathrm{e}^{-} .
$$

This reaction has a low energy threshold: Epor $=0.23 \mathrm{MeV}$, thus, the neutrinos are recorded from the main reaction

$$
\mathrm{p}+\mathrm{p} \rightarrow 2 \mathrm{H}+\mathrm{e}+v \mathrm{e},
$$

in which the neutrino emission is highest $(77 \%$ ve). The gallium-germanium method is implemented in the laboratory located in Baksan beneath the Cheget mountain where there are several tons of gallium in which the neutrino reaction with gallium occurs. Neutrino detection can also be conducted by the lithium method and others. Table 2 illustrates the comparative efficiency of these methods in the detection of the different neutrino types.

The highest detection efficiency of boron neutrinos is implemented in the chlorine-argon method (37C1); pp-neutrinos are most efficiently detected by the gallium-germanium method $(71 \mathrm{Ga})$, and pep-neutrinos, by the lithium method (7Li).

All the detectors use a huge working body: for example, the chlorine-argon method uses a tank with a volume of $400 \mathrm{~m}$ filled with chlorine-containing substance (610 tons of C2Cl4) which is placed deep underground. The $37 \mathrm{Ar}$ atoms were dissolved in the total mass of the detector $\mathrm{M}=21,030$ atoms. The gaseous argon atoms produced in the reaction were passed through the detector which had a size of $5 \mathrm{~cm}^{3}$. Our task was to register $\sim 60$ atoms during 3 - 4 months - the time of the solar neutrino detection experiment. The implementation of the gallium-germanium method requires 60 tons of gallium. Previously it was hypothesized that the radioactive materials have much larger cross section of interaction with neutrinos [25]. How can it be proven? A well known fact is that neutrinos are the product of the weak interaction and it is likely that they should have the maximal cross section of interaction with neutrinos exactly in the beta-decay of radioactive materials. 137Cs is such a radioactive material. Cesium-137 is a daughter product of $\beta$-decay of the 137Xe nuclide (with a half-life of 3.818 (13) [2] min): 137Xe54 $\rightarrow 137 \mathrm{Cs} 55+\mathrm{e}^{-}+$ve. Cesium-137 undergoes beta decay (the half-life is 30.17 years), which produces a stable barium isotope 137Ba:137Cs55 $\rightarrow{ }^{137} \mathrm{Ba} 56+\mathrm{e}^{-}+v_{\mathrm{e}}$.

The decay in 94.4\%, occurs with the intermediate formation of the nuclear isomer of barium-137, 137Bam (a half-life is $2.55 \mathrm{~min}$ ), which, in turn, passes into the ground state with the emission of a gamma-ray with an energy of $661.7 \mathrm{keV}$ (or a conversion electron with an energy $661.7 \mathrm{keV}$ minus the electron-binding energy). The total energy released in the beta decay of one nucleus of Cesium -137 is $1175.63 \pm 0.17$ [1] keV.

Thus, we can detect the gamma radiation emitted by the decay of cesium. On the other hand, in the decay chain of uranium, the intermediate member-radium B-is formed. This member also undergoes beta decay with a half-life of 26.8 minutes and $1.024 \mathrm{MeV}$ energy release. If a neutrino interacts with the beta-decay isotopes [2]

Table 2. Efficiency of the methods for neutrino detection.

\begin{tabular}{ccccc}
\hline Neutrino Type & $37 \mathrm{C} 1$ & $7 \mathrm{Li}$ & 0 & $71 \mathrm{Ga}$ \\
pp & 0 & $26 \%$ & $0.4 \%$ \\
pep & $4.5 \%$ & $15 \%$ & $19 \%$ \\
${ }^{7} \mathrm{Be}$ & $16 \%$ & $43 \%$ & $1 \%$ \\
${ }^{8} \mathrm{~B}$ & $76 \%$ & $16 \%$ & $3 \%$ \\
${ }^{13} \mathrm{~N}$ & $3.5 \%$ & 3 & 3 \\
\hline
\end{tabular}


[3], then, when synchronously recording the variations in the intensity of radio activity of the cesium and uranium decay products, we should obtain a time shift in the registration of the neutrino outbursts. We carried out the described experiment.

As a continuation of these studies, we conducted synchronous measurements of gamma variations of the ${ }^{137} \mathrm{Cs}$ cesium isotope and Cisbaikalian uranium ore. The 137Cs itself is beta-active; however, the resulting barium 137Ba gives monochromatic gamma rays at energies of ${ }^{137} \mathrm{Cs} \rightarrow 661 \mathrm{keV}$. The uranium ore Ur placed in a closed metal container decays into the radioactive isotopes including radium, radon and its decay isotope products, which are in equilibrium. Synchronous recording of $\gamma$-variation cesium Cs137 and Ur ore was conducted by the SBM 19 Geiger type radiation detectors. Data recording was carried out by the digital microprocessorcontrolled E-clerk device with a sampling frequency of 2 minutes and then transferred to a personal computer, i.e. we used the previous registration scheme [2] [3]. As a result, we obtained two synchronous realizations (time series) of the variations in $\gamma$ radioactivity of cesium and ore with a duration of more than 13 days. Next, we averaged these time series over 4-min intervals and calculated the cross-correlation function (CCF) between them. Figure 1 shows a fragment of CCF between the time series of cesium (Cs137) and uranium ore (Ur).

As the figure shows, the maximum in CCF has a lag of 28 minutes. Next, we calculated the moving correlation in a $1 \%$ window between the time series of variations in $\gamma$ radioactive cesium 137Cs and $\gamma$ radioactivity of uranium ore Ur with a 28-min lag and obtained the sliding correlation function (SCF) between them. This function is shown in Figure 2.

The $1 \%$ window was selected in order to provide sufficient statistics for determining the significance of the coefficients of correlation. The total number of the independent values of CSF is above 4500 and, hence, a $1 \%$ window values contained more than 45 values (points), which is quite enough for the significant estimation of reliability (Figure 2). The analysis of CSF shows that within some intervals with a duration of a few hours (there are at least three such intervals), we obtain the correlation coefficients $\mathrm{k}>0.6-0.7$ with the significance $P>0.99$. This means that with a probability $P>0.99$ there is a correlation between these variations in $\gamma$ radioactivity. However, this indicates that radioactivity of cesium and uranium ore is affected by the third agent. Table 3 shows the results of spectral analysis for the synchronous variations in $\gamma$ radioactive cesium 137 (column 2) and Baikalian uranium ore (column 3) compared to the periods of the free oscillations of the Sun predicted by the theory (column 5). Column 4 indicates the spectral peaks of CCF between the cesium and uranium ore. Column 6 presents the modes and forms of the free oscillations of the Sun.

In Table 3, the significant $(\mathrm{P}>0.99)$ spectral peaks coinciding with the theoretical solar data up to three signs inclusive [3] are indicted in the boldface. Thus, we can conclude that the main agent affecting the gamma radioactivity of Cs137 and uranium ore is the Sun in the form of the neutrino variations arising in the fusion reactions which are modulated by the free oscillations of the Sun and registered by our detectors consisting of a conventional Geiger counters recording the gamma radiation from the nearby radioactive sources.

In this case, our neutrino detector works as follows: 1) neutrinos are captured by the radioactive isotope and their gamma radiation increases; 2) an ordinary SBM 19 Geiger type counter or other counter records this increase of the radiation; 3 ) the difference between the normal radioactivity and the radioactivity when exposed to

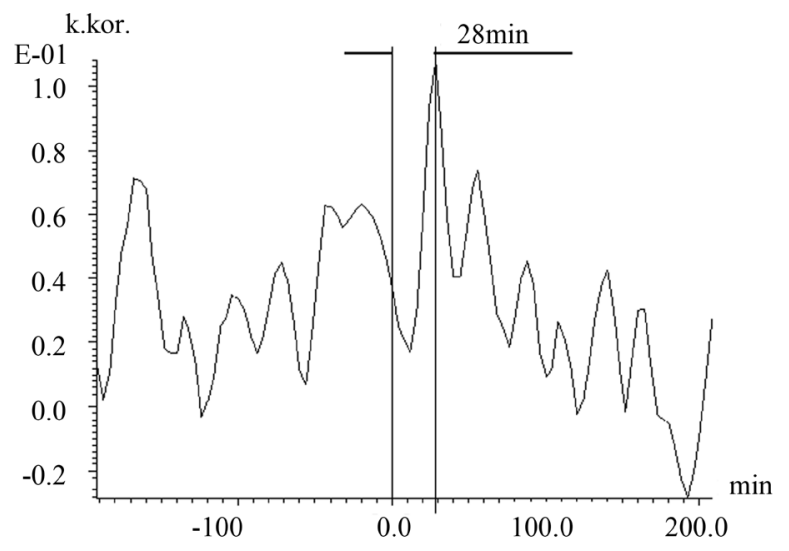

Figure 1. The fragment of the cross-correlation function. 


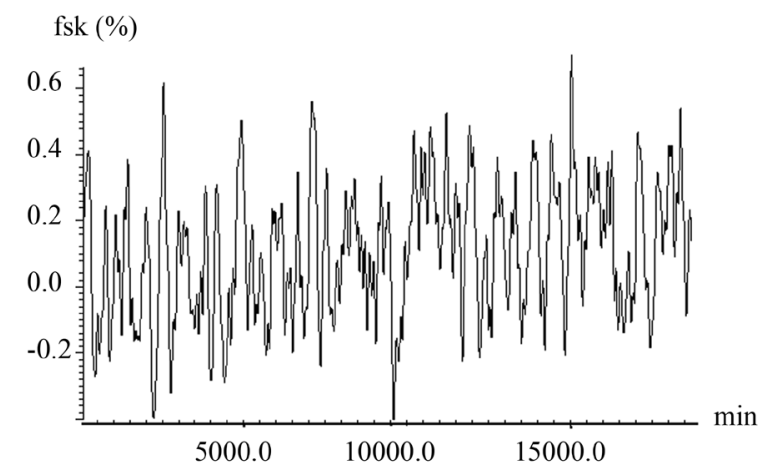

Figure 2. The sliding correlation function (CSF) between the variation of Cs137 and Ur ore.

neutrino impact is the sought effect.

The experiments with the neutrino influence on the isotopes were carried out and a positive effect was obtained (see above). For data transferring by the neutrino beam, the latter should have a sufficiently high modulation frequency. This possibility is provided by the Tevatron neutrino beam accelerator in the Fermi laboratory near Chicago. The record was achieved eight years ago: the modulation frequency of the neutrino beam was 26Gts. However, less expensive technologies and more mobile systems are required on this way.

\section{New Principles of the Neutrino Beam Emitters}

The standard neutrino sources are the nuclear beta decay reactions. The neutrino beams obtained in the protons-muons-neutrinos accelerators are very cumbersome however suitable for producing very narrow beams ( 2 $\mathrm{mm}$ ). Table 2 shows the relative efficiency of these methods in detecting different neutrino types. The highest detection efficiency of boron neutrinos is implemented in chlorine-argon method (37C1). The pp-neutrinos are most efficiently detected by the germanium gallium (71Ga) method, and the pep-neutrino, by the lithium method (7Li). Table 4 presents the beryllium isotopes whose irradiation by an electron beam ban produce a $+7 \mathrm{Li}$ neutrino beam according to the B. Pontecorvo reversibility principle.

The neutrino beam obtained in this way will probably be less efficient than the beam produced in the classical way at the accelerator. However, this method does not require huge accelerators and is very compact. The entire installation for producing a neutrino beam is largely determined by the dimensions of rather small betatron. Except for the beryllium-lithium isotopes, neutrino beams can also be produced by the germanium-gallium method. Table 5 presents the germanium isotopes whose irradiation by an electron beam also yields a $+71 \mathrm{Ga}$ neutrino beam according to the principle of reversibility of Pontecorvo. Table 6 presents the other radionuclides.

Thus, we considered the new schemes for the formation of the neutrino beams based on the new principles and materials.

\section{Solar Neutrinos and Other Particles [27] [28]: The Search Experiments and the Monopole}

According to the Dirac version of quantum theory, interaction between the electric charge e and a magnetic charge $g$ is possible if $\frac{e g}{2 \pi \hbar c}=n$, where $\mathrm{n}$ is an integer. This means that the magnetic charge of a particle should be a multiple of the elementary magnetic charge $g_{0}=\frac{2 \pi \hbar c}{e}$, where $e$ is the elementary electric charge.

In this case, the existence of the magnetic charge (monopole) is only consistent with the standard quantum mechanics if the electrical charges of all the particles are quantized. A particle which possesses both the electric and magnetic charges is defined as a dyon. According to the Hooft-Polyakov model, the interaction with the magnetic monopole can initiate the decay of the nucleon predicted by some models of grand unification, that is, to act as a catalyst for this decay. The Dirac's theory does not predict the mass of the magnetic monopole, a consensus in the assessment of the monopole mass is absent. Unsuccessful experiments on monopole detection 
Table 3. The results of spectral analysis of synchronous variations in the radioactive elements comparison with the periods of natural oscillations of the Sun [26]. p, g, f are the modes of the free oscillations of the Sun; 1 is the shape of the free oscillations.

\begin{tabular}{|c|c|c|c|c|c|}
\hline $\mathrm{N}$ & $\begin{array}{l}\text { Cs137 (h, min) } T(\mathrm{~h}, \min ) \\
\text { Observ. }\end{array}$ & $\begin{array}{l}\text { Bur (h, min) } \\
\text { T (h, min) } \\
\text { Observ. }\end{array}$ & $\begin{array}{c}\mathrm{Cs}_{137} \times \mathrm{BUr} \\
T(\mathrm{~h}, \mathrm{~min}) \\
\text { observ. }\end{array}$ & $\begin{array}{l}\text { FSO } \\
\text { Ts (min) } \\
\text { (theor.) }\end{array}$ & $\begin{array}{l}\text { Note } \\
\text { (mode) } p, g, f \text {-modes }\end{array}$ \\
\hline 1 & $136.5 \mathrm{~h}$ & $136.5 \mathrm{~h}$ & $136.5 \mathrm{~h}$ & & \\
\hline 2 & 30.3 & & & & \\
\hline 3 & 21.0 & 21.0 & & & \\
\hline 4 & & 9.9 & & & \\
\hline 5 & & 7.8 & & & \\
\hline 6 & $5.46 \mathrm{~h}(327.6 \mathrm{~m})$ & 6.07 & & & \\
\hline 7 & $215.4 \mathrm{~m}$ & $212.8 \mathrm{~m}$ & $215.4 \mathrm{~m}$ & & \\
\hline 8 & & 176.2 & 176.2 & $175.9 \mathrm{~m}$ & $g 16, l 3$ \\
\hline \multicolumn{6}{|l|}{9} \\
\hline 10 & & 117.0 & & 118.9 & $g 10.13$ \\
\hline 11 & 110.7 & & & 109.7 & $g 8, l 3$ \\
\hline 12 & & 103.7 & 100.5 & 100.7 & $g 8, l 3$ \\
\hline 13 & 86.2 & 87.1 & 87.1 & 84.4 & $g 2, l 1$ \\
\hline 14 & 81.1 & 73.1 & 75.8 & $74.9 ; 76.8$ & $g 5, l 3 ; g 7, l 4$ \\
\hline 15 & 61.8 & 63.8 & & 62.29 & $p 1, l 1$ \\
\hline 16 & & 55.9 & 53.7 & $57.3 ; 57.73$ & $p 1, l 2 ; g 3, l 4$ \\
\hline 17 & 49.5 & 50.7 & 49.6 & 49.59 & $g 2,14$ \\
\hline 18 & 42.1 & 42.7 & 42.2 & 44.18 & $g 1,14$ \\
\hline 19 & 40.16 & & 40.16 & 40.97 & $f, l 3$ \\
\hline 20 & 38.8 & & 38.7 & 38.82 & $f, 14$ \\
\hline 21 & 37.6 & 36.3 & 36.3 & 36.98 & $p 2, l 1$ \\
\hline 22 & 35.4 & & 35.5 & 37.58 & $p 1,14$ \\
\hline 23 & 33.5 & & 33.5 & & \\
\hline 24 & 32.7 & 32.0 & 32.4 & 32.19 & $p 2, I 2$ \\
\hline 25 & & 31.5 & 31.5 & 30.93 & $p 3,10$ \\
\hline 26 & 30.62 & 30.1 & 30.5 & 30.93 & $p 3,10$ \\
\hline 27 & 28.05 & 28.05 & 28.05 & 27.88 & $p 3, l 1$ \\
\hline 28 & & 26.5 & 26.5 & 27.62 & $p 2,14$ \\
\hline 29 & 25.2 & 25.4 & & 25.09 & $p 3,12$ \\
\hline 30 & & 24.3 & 24.5 & 24.49 & $p 4,10$ \\
\hline 31 & 23.8 & 23.9 & 23.9 & 23.21 & $p 3, l 4$ \\
\hline 32 & 22.3 & 22.1 & 22.1 & 22.30 & $p 4,12$ \\
\hline 33 & 21.8 & & & 21.92 & $p 3, l 4$ \\
\hline 34 & 20.35 & 20.3 & 20.3 & 20.52 & $p 4, I 2$ \\
\hline 35 & 18.8 & 18.9 & 18.9 & 19.26 & $p 4, l 3$ \\
\hline 36 & 18.6 & 18.6 & 18.6 & 18.68 & $p 4, I 1$ \\
\hline 37 & 18.2 & & & 18.31 & $p 4,14$ \\
\hline 38 & 17.17 & & & 17.17 & $p 6,10$ \\
\hline
\end{tabular}


Table 4. Beryllium isotopes used for the creating the neutrino beam by the Pontecorvo method.

\begin{tabular}{|c|c|c|c|c|c|}
\hline $\begin{array}{c}\text { The sym } \\
\text { bol }\end{array}$ & $\begin{array}{l}\text { Z (p) } \\
\text { N (n) }\end{array}$ & $\begin{array}{c}\text { Isotope mass, } \\
\text { (A.E.M.) The excitation } \\
\text { energy }\end{array}$ & $\begin{array}{l}\text { Half life } \\
\text { [2] (T1/2) }\end{array}$ & $\begin{array}{l}\text { Decay modes } \\
\text { Of the nucleus }\end{array}$ & Spin and parity \\
\hline $5 \mathrm{Be}$ & $\begin{array}{l}4 \\
1\end{array}$ & $5.04079(429)$ & & $\mathrm{p}$ & $(1 / 2+) \#$ \\
\hline 7Be & $\begin{array}{l}4 \\
3\end{array}$ & $7.01692983(11)$ & 53.22 (6) сут & $\mathrm{EC}$ & $3 / 2-$ \\
\hline 10Be & $\begin{array}{l}4 \\
6\end{array}$ & $10.0135338(4)$ & $1.51 \times 10^{6}$ year $[6.8(17) \mathrm{eV}$ & $\beta-$ & $0+$ \\
\hline
\end{tabular}

Table 5. Germanium isotopes applicable for producing the neutrino beam by the Pontecorvo method.

\begin{tabular}{ccccc}
\hline The symbol & $\begin{array}{c}\mathrm{Z}(\mathrm{p}) \\
\mathrm{N}(\mathrm{n})\end{array}$ & $\begin{array}{c}\text { Isotope mass, } \\
\text { (A. E. M.) The excitation energy }\end{array}$ & $\begin{array}{c}\text { Half life } \\
{[2](\mathrm{T} 1 / 2)}\end{array}$ & Spin and parity \\
\hline $68 \mathrm{Ge}$ & 32 & 67.928094 & 270.95 day & $0+$ \\
& 36 & & & $5 / 2-$ \\
$69 \mathrm{Ge}$ & 32 & 68.9279645 & 39.05 our & $1 / 2-$ \\
$71 \mathrm{Ge}$ & 37 & 70.9249510 & 11.43 day & $9 / 2+$ \\
\hline
\end{tabular}

Table 6. Other radio nuclides with beta-decay.

\begin{tabular}{|c|c|c|c|c|c|}
\hline \multirow{2}{*}{ Radionuclide } & \multicolumn{2}{|c|}{ The specific activity of the isotope $100 \%$} & \multirow{2}{*}{ Half life } & \multirow{2}{*}{$\begin{array}{c}\text { Type of } \\
\text { decay }\end{array}$} & \multirow{2}{*}{$\begin{array}{c}\text { Energy } \\
(\mathrm{max}) \\
{[\mathrm{MeV}]}\end{array}$} \\
\hline & {$[\mathrm{mCi} / \mathrm{mmol}]$} & {$[\mathrm{Bk} / \mathrm{mole}]$} & & & \\
\hline 3H (tritium) & 29.05 & $1.11 \times 10^{15}$ & $12.43 \mathrm{y}$ & $\beta$ & 0.0185 \\
\hline $14 \mathrm{C}$ & 0.062 & $2.3 \times 10^{12}$ & $5730 \mathrm{y}$ & $\beta$ & 0.156 \\
\hline $32 \mathrm{P}$ & 9104 & $0.33 \times 10^{18}$ & $14.3 \mathrm{~d}$ & $\beta$ & 1.709 \\
\hline $33 \mathrm{P}$ & 5138 & $0.19 \times 10^{18}$ & $25.4 \mathrm{~d}$ & $\beta$ & 0.249 \\
\hline $35 \mathrm{~S}$ & 1491 & $0.5 \times 10^{17}$ & $87.4 \mathrm{~d}$ & $\beta$ & 0.167 \\
\hline $125 \mathrm{I}$ & 1672 & $0.8 \times 10^{17}$ & $60 \mathrm{~d}$ & e.c. & 0.25 \\
\hline
\end{tabular}

have been conducted during several decades. Different interaction models were assumed in these experiments. A heavy magnetic monopole should have a high penetrating power and create strong ionization along its path. Also the magnetic monopoles trapped in the magnetic ores of terrestrial and extraterrestrial origin as well as the tracks left by them in mica enclosed in the ancient terrestrial rocks were sought for. On the other hand, we note the experiments on the search for dark matter (Prof. George Fraser, Monthly Notices of the Royal Astronomical Society, October, 2014); and search for axions-the dark matter particles (University of Leicester). A team of the researchers believes that axions, the candidates for the dark matter particles, are generated in the core of the Sun and are indeed transformed into the X-ray radiation in the Earth's magnetic field. In the opinion of some scientists, neutrinos can also be converted into monopoles under the passage of the powerful magnetic field. Scientists at the Purdue University have found that the decay rate of a radioactive sample has slightly changed 39 hours before the onset of a large solar flare. The similar changes were revealed in the decay rate before and after the solar flares as well as in the Earth's rotation around the Sun, the rotation of the Sun, and solar activity variations. In this case, the annual fluctuations have a simple explanation: when the Earth is located farther from the Sun, it receives less solar neutrinos and the decay rate slows down to a certain extent. When the Earth approaches the Sun or when a solar flare occurs, the number of neutrinos grows and the decay accelerates. It is also suggested that the fluctuations in the decay rate can be caused by the other, unknown particles. 
Based on the studies [2]-[5] and the said, we consider yet another signal detection method which has been in fact used in the analysis of the neutrino flux from the Sun and was previously applied in the study of the gas-dust flows [29]. As is known, in astrophysics and astronomy, various fields and particles are studied by the corpuscular and wave methods. For example, the cosmic dust was initially investigated by the corpuscular method as the particles of the matter. These studies determined the chemical composition, particle size, etc. Next, a different approach was applied: dust (particles) was treated as an ensemble or a volume of gas or dust plasma. This approach precluded from considering the individual properties and composition of the particles but allowed identifying the cooperative, wave features of dust and gas flow, which were previously unknown, through the variations in the parameters of the testing process (in this case, seismic) [29]. This approach enabled the scientists to discover the modulation of gas and dust streams at the periods of the orbital and free rotation of celestial bodies. The similar method was applied in [2] [3] where the signal detection was based on the criterion other than the occurrence of the event of inelastic neutrino interaction with target material, which underlies the modern methods of registration. Instead, the signal in the cited works was detected by the appearance of typically hidden time periodicities in the level of the test process (usually the activity of the $\gamma$-source), which are initially only characteristic of the source of the neutrino flux. The similar approach resulted in the discovery of the effect of anomalously high cross section of neutrino capture by heavy radioactive nuclei and, simultaneously, the modulation effect of the solar neutrino flux by the spectrum of solar oscillations (the latter is quite obvious) [2] [3]. Since, according to the assumption of many experts, the oscillations of the central zone of the Sun is accompanied by the generation of the other elementary particles as well whose existence still requires experimental validation (detection), for example, monopole, the search for such particles was carried out by the modified experimental scheme [2] [3], Figure 3.

As previously, the experiments on monitoring and studying the probable elementary particles of solar origin were conducted by measuring the variations in the probable gamma radiation in the container 1 (Figure 3) which, in contrast to the previous measurement scheme, did not contain a radioactive source and is only permeable for the expected or existing particles (neutrinos, monopoles ). The measurements were conducted with the independent EClerk digital recorder with 1-min sampling of the detecting device (radiometer) and the subsequent data transferring on the computer by the scheme (Figure 3).

Correspondingly, the sealed steel container with a volume of $0.5 \mathrm{l}$ was only permeable for the background gamma-radiation and the expected particles (see above) whose interactions with the magnetic field were registered by the radiation detector (SBM19 Geiger type) (2), and the obtained value o was every minute recorded on the memory of the autonomous "EClerk” DVR (3). The data were then processed on the personal computer (4). The radiometer was placed into a sealed steel container 1 together with the magnets. This limited the effects of atmospheric pressure (the pressure variations barely affected the ore (we mean here the amount of radon as a decay product)) and the background radiation. The signals were recorded in the magnetic field in 2 configurations A, B (Figure 4). The container was penetrated by the background cosmic radiation, both known (the solar and cosmic neutrinos) and presumed (monopole, dark matter, etc.).

Using this installation (Figure 3 and Figure 4), we recorded a number of the observations whose shapes resemble the time series of radioactivity [2]-[5]. The series was then subjected to spectral analysis, and the reliable values of the spectral peaks $(\mathrm{P}>95)$ were compiled into Table 7 .

From the analysis of Table 7 it follows that the periods are generally consistent with the values of the tables

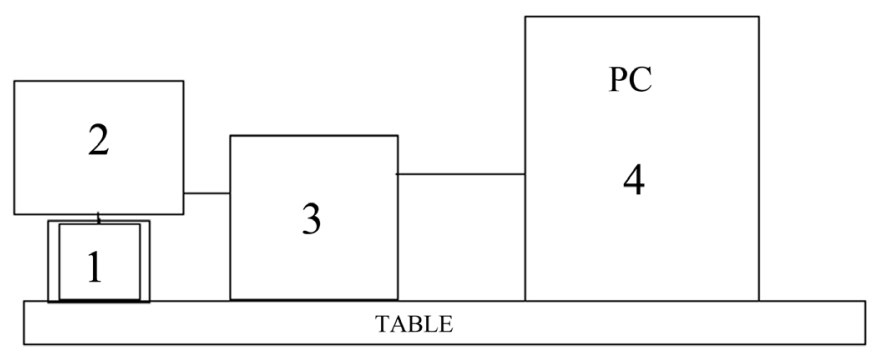

Figure 3. The scheme of the search for the variations in the particles of solar origin. 1-Container; 2-Radiation detector; 3-Digital recorder; 4-Personal computer. 

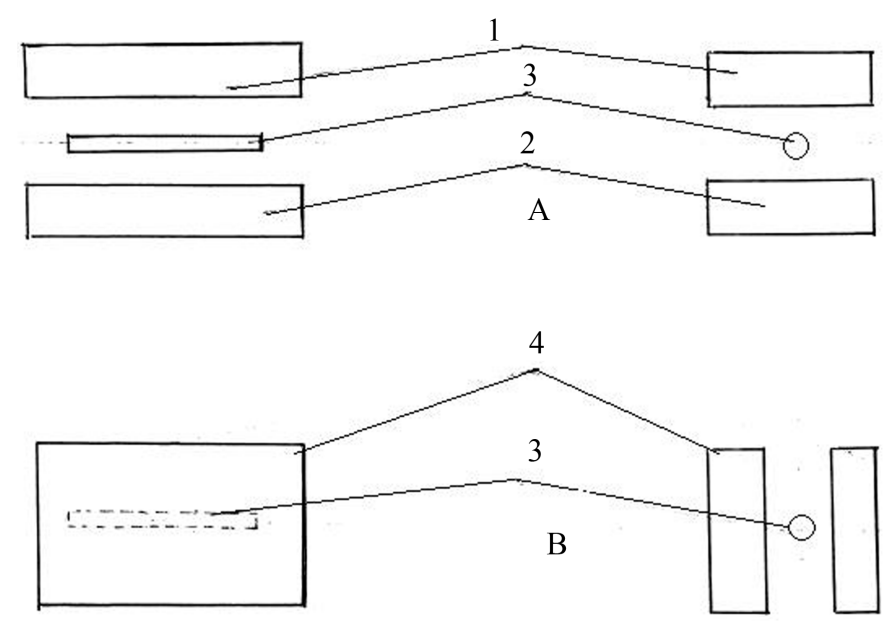

Figure 4. The scheme of the container 1 (Figure 3) for searching and/or studying the elementary particles of solar origin. A, B: the setup configurations of the permanent powerful magnet blocks with a size of $12 \times 8 \times 1.5 \mathrm{~cm}$ with a strong magnetic field and $\mathrm{H}>100 \mathrm{G}$ or with $108 \mathrm{G}$ with the domain structure of the magnets taken into account. A: 1, the horizontal top magnet, 2, the horizontal bottom magnet, 3-SBM-19 Radiometer; B: 4, vertical magnets; 3, radiometer.

in [2]-[5] [20], but their coincidence with the periods of solar oscillations is not as impressive as in [2]-[5] [20]. By examining the data (moving along the spectrum from the short to the long periods) one can see that there are groups with a good coincidence $(\sim 1 \%-3 \%)$ and a worse coincidence $(4 \%-5 \%)$. The cause of this is unclear. One of the probable mechanisms is a stronger modulation of the oscillations by the amplified magnetic field. Generally, we should note the existence of the forms of interaction between the elementary solar particles (neutrinos, monopole, etc.) and the strong magnetic field, which is accompanied by the $\gamma$-radiation affecting the radiometer.

In this case, this effect should also be observed in the megaworld. For example, the negative correlation between the solar activity (Wolf numbers) and neutrino fluxes as well as the correlation of seismic activity with the variations in the high-energy neutrino flow is revealed [30]-[32]. Indeed, under the strong solar activity, the magnetic fields become intensified and turbulized, which leads, according to the results of the experiment, to the absorption of elementary particles. With the anomalously high local density of the magnetic field ( $10^{4}$ gauss), according to the experts, the "explosion of the magnetic field" (a solar flare) occurs, that is, the flow from the inner regions of the Sun (for the neutrinos $4 \times 1014 \mathrm{sm}^{2}$ ) reaches and interacts with the field concentration zone, which is accompanied by the release of significant energy ( $1025 \mathrm{~J}$ for a flare). The results obtained by the high-altitude balloons telescope (Kiruna, Sweden, 2014) contradict the explosion of the magnetic field. Here, an unknown process takes place: the hottest points on the surface of the Sun are not dark but white; they are scattered across the surface and it is likely that a flare occurs at the time of their spontaneous integration.

The connection between the magnetic storms on the Earth or on a regional scale and the local seismicity is equally interesting.

\section{Conclusions}

1) The sensitivity of the neutrino sensor is estimated (tested).

2) The principles of neutrino detection are analyzed.

3) The new principles of the neutrino beam emitters are suggested.

4) The pilot experimental setup for studying the interaction between the elementary particles of solar origin (neutrinos, monopole) and a powerful magnetic field is developed.

5) The interaction between the solar particle flux of (neutrinos, monopole, etc.) with the strong magnetic field is detected. This interaction is accompanied by the $\gamma$-radiation acting on the radiometer. As was previously observed, this flux is modulated at the periods of the free oscillations of the Sun. 
Table 7. The observed significant $(\mathrm{P}>0.95)$ periods in the spectrum of the background intensity variations of cosmic radiation in the strong $\mathrm{H}>100 \mathrm{Gs}$ or $108 \mathrm{G}$ (taking into account the domain structure of magnets) magnetic field.

\begin{tabular}{|c|c|c|c|}
\hline № & $\mathrm{T}(\mathrm{h}, \mathrm{min})$ & $\mathrm{T}_{\mathrm{T}}(\mathrm{min})$ & Note \\
\hline 1 & $136.5 \mathrm{~h}$ & & \\
\hline 2 & $24.3 \mathrm{~h}$ & & \\
\hline 3 & $12.0 \mathrm{~h}$ & & \\
\hline 4 & $5.57 \mathrm{~h}$ & & \\
\hline 5 & $4.7 \mathrm{~h}$ & & \\
\hline 6 & $3.33 \mathrm{~h}$ & & \\
\hline 7 & $142.2 \mathrm{~min}$ & 141.5 & $g 16, l=4$ \\
\hline 8 & 94.2 & 91.8 & $g 7, l=3$ \\
\hline 9 & 71.4 & 70.3 & $g 6, l=4$ \\
\hline 10 & 66.6 & 64.89 & $g 5, l=4$ \\
\hline 11 & $58.3 \mathrm{~min}$ & 57.73 & $g 3, l=4$ \\
\hline 12 & 55.0 & 55.05 & $g 1, l=2$ \\
\hline 13 & 51.2 & & \\
\hline 14 & 50.1 & & \\
\hline 15 & 48.8 & & \\
\hline 16 & 47.2 & 47.94 & $g 1, l=3$ \\
\hline 17 & 45.8 & 45.9 & $f, l=2$ \\
\hline 18 & 43.4 & & \\
\hline 19 & 42.9 & 42.5 & $p 1, l=2$ \\
\hline 20 & 38.4 & 38.82 & $f, l=4$ \\
\hline 21 & 36.7 & 36.98 & $p 2, l=2$ \\
\hline 22 & 34.5 & & \\
\hline 23 & 32.4 & & \\
\hline 24 & 31.5 & & \\
\hline 25 & 30.7 & 30.93 & $p 3, l=0$ \\
\hline 26 & 29.6 & & \\
\hline 27 & 29.1 & & \\
\hline 28 & 25.2 & 25.09 & $p 4, l=2$ \\
\hline 29 & 24.3 & 24.49 & $p 4, l=0$ \\
\hline 30 & 23.5 & 23.21 & $p 3, l=3$ \\
\hline 31 & 22.9 & & \\
\hline 32 & 22.1 & 22.30 & $p 4, l=1$ \\
\hline 33 & 21.7 & & \\
\hline 34 & 21.4 & & \\
\hline 35 & 20.6 & 20.52 & $p 4, l=2$ \\
\hline 36 & 20.4 & & \\
\hline 37 & 19.95 & & \\
\hline 38 & 19.5 & 19.26 & $p 4, l=3$ \\
\hline
\end{tabular}




\section{Continued}

\begin{tabular}{|c|c|c|c|}
\hline 39 & 18.0 & & \\
\hline 40 & 17.3 & 17.39 & $p 5, l=2$ \\
\hline 41 & 14.9 & 14.93 & $p 7, l=0$ \\
\hline 42 & 14.3 & & \\
\hline 43 & 14.1 & 14.08 & $p 7, l=1$ \\
\hline 44 & 13.8 & & \\
\hline 45 & 13.3 & 13.35 & $p 7, l=2$ \\
\hline 46 & 13.2 & 13.21 & $p 8, l=0$ \\
\hline 47 & 12.9 & & \\
\hline 48 & 12.8 & 12.77 & $p 7, l=3$ \\
\hline 49 & 12.1 & & \\
\hline 50 & 11.7 & & \\
\hline 51 & 11.5 & 11.51 & $p 8, l=3$ \\
\hline 52 & 11.4 & & \\
\hline 53 & 11.3 & & \\
\hline 54 & 11.2 & & \\
\hline 55 & 11.1 & & \\
\hline 56 & 10.4 & 10.49 & $p 9, l=3$ \\
\hline 57 & 9.8 & & \\
\hline 58 & 9.5 & 9.54 & $p 11, l=1$ \\
\hline
\end{tabular}

6) It is also necessary to consider the model of a solar flare based on identifying the flows of elementary particles from the central zone of the Sun in the zone of the energy outburst.

\section{References}

[1] Khavroshkin, O. and Tsyplakov, V. (2011) Radioactivity of Nuclei in a Centrifugal Force Field. The Natural Science (NS), 3, 733-737.

[2] Khavroshkin, O. and Tsyplakov, V. (2013) Sun, Earth, Radioactive Ore: Common Periodicity. The Natural Science (NS), 5, 1001-1005.

[3] Khavroshkin, O.B. and Tsyplakov, V.V. (2013) The Radioactivity, Solar Neutrino, Interactions. Engineering Physics, Moscow, No. 8, 53-62.

[4] Khavroshkin, O.B. and Tsyplakov, V.V. (2013) Radioactivity Ore Sample: Monitoring. Engineering Physics, 53-61.

[5] Khavroshkin, O.B. and Tsyplakov, V.V. (2013) Natural Radioactivity as an Open System. Engineering Physics, 40-54.

[6] Falkenberg E.D. (2001) Radioactive Decay Caused by Neutrino? Apeiron, 8, 32-45.

[7] Siegert, H., Shrader, H. and Schotzis, U. (1998) Half-Life Measurements of Europium Radio Nuclides and the Long-Term Stability of Detectors. Applied Radiation and Isotopes, 49, 1397-1401.

[8] Ellis, K.J. (1990) The Affective Half-Life of a Broad Beam ${ }^{238}$ PuBe Total Body Neutron Irradiator. Physics in Medicine and Biology, 35, 1079-1088.

[9] Alburder, D.E., Harbottle, G. and Norton, E.F. (1986) Half-Life of ${ }^{32}$ Si. Earth and Planetary Science Letters, 78, 169.

[10] Parkhomov, A.G. and Maklyaev, E.F. (2004) Search of Rhyhmtms and Fluctuations of Radioactivity, Quartzes Resonators, Noise of Half Conductivity, Temperature and Atmosphere Pressure in Long Time Measurements. Physical Thought of Russia, No. 1, 1-12.

[11] Parkhomov, A.G. (2010) Researches of Alpha Beta Radioactivity at Long-Term Observations. arXiv:1004.1761v1 [physics.gen-ph].

[12] Parkhomov, A.G. (2009) Space. Earth. Human. New Aspects of Science. Science, Moscow. 
[13] Baurov, Y.A., Sobolev, Y.G., et al. (2000) Experimental Research of Change Velocity of Beta Decay for Radioactivity Elements. Physical Thought of Russia, No. 1, 1-7.

[14] Baurov, Y.A. and Malov, I.F. (2010) Variations of Decay Rates of Radio-Active Elements and Their Connections with Global Anisotropy of Physical Space. arXiv:1001.5383v1 [physics.gen-ph].

[15] Sturrock, P.A., Buncher, J.B., Fischbach, E., Gruenwald, J.T., Javorsek II, D., Jenkins, J.H., Lee, R.H., Mattes, J.J. and Newport, J.R. (2010) Power Spectrum Analysis of Physicalisch-Technische Bundesanstalt Decay-Rate Data: Evidence for Solar Rotational Modulation. Solar Physics, 267, 251-265. http://dx.doi.org/10.1007/s11207-010-9659-4

[16] Jenkins, J.H., Herminghuysen, K.R., Blue, T.E., Fischbach, E., Javorsek II, D., Kauffman, A.C., Mundy, D.W., Sturrock, P.A. and Talnagi, J.W. (2012) Additional Experimental Evidence for a Solar Influence on Nuclear Decay Rates. Astroparticle Physics, 37, 81-88. http://dx.doi.org/10.1016/j.astropartphys.2012.07.008

[17] Parkhomov, A.G. (2009) Research Alpha and Beta Radioactivity of Long-Term Measurements. Space. Earth. Human. New Aspects of Science. Science, Moscow.

[18] Sturrock, P.A., Parkhomov, A.G., Fischbach, E. and Jenkins, J.H. (2012) Power Spectrum Analysis of LMSU (Lomonosov Moscow State University) Nuclear Decay-Rate Data: Further Indication of R-Mode Oscillations in an Inner Solar Tachocline. Astroparticle Physics, 35, 755-758. http://dx.doi.org/10.1016/j.astropartphys.2012.03.002

[19] Parkhomov, A.G. (2011) Deviations from Beta Radioactivity Exponential Drop. Journal of Modern Physics, 2, 13101317. http://dx.doi.org/10.4236/jmp.2011.211162

[20] Starodubov, A.V., Khavroshkin, O.B. and Tsyplakov, V.V. (2014) From the Periodicity of Radioactivity to the Cosmic and Metaphysical Oscillations. Metaphysics, No. 1, Russian Peoples’ Friendship University, Moscow, 137-149.

[21] Shestopalov, I.P., Belov, S.V., Soloviev, A. A. and Kuzmin, Y.D. (2013) About Neutron Generation and Geomagnetic Disturbance which Connection with Chilean Earthquake on 27 February and a Volcanic Eruption in Iceland in MarchApril 2010. Geo Magnet and Aeronomic, 53, 130-142.

[22] Wang, H.-Z. (1990) On the Internal Energy Source of the Large Planets. Chinese Astronomy and Astrophysics, 14, 361-370. http://dx.doi.org/10.1016/0275-1062(90)90015-6

[23] Letnikov, F.A. (2001) On the Problem of the Earth's Internal Heat Source. Reports of the Russian Academy of Sciences, 378, 387-389.

[24] Anisichkin, V. F., Voronin, D.V. and Kryukov, B.P. (1999) Calculation of the Fragmentation of the Planets in the Explosion. Proceedings of the International Conference, V Zababakhin Scientific Readings, VNIITF Publisher, Snezhinsk, 89-91.

[25] Dodonov, V.V., Klimov, A.B. and Man’ko, V.L. (1996) Low Energy Wave Packet Tunneling from a Parabolic Potential Well through a High Potential Barrier. Physics Letters A, 220, 41-48. http://dx.doi.org/10.1016/0375-9601(96)00482-3

[26] Iben Jr., I. and Mahaffy, J. (1976) On the Sun’s Acoustical Spectrum. Astrophysical Journal, 209, L39-L43. http://dx.doi.org/10.1086/182262

[27] Bolotovski, B.M., Yu, D. and Usachev, M. (Eds.) (1970) Dirac Monopole. Collection of Articles, Translated from English.

[28] Polyakov, A.M. (1974) Particle Spectrum in the Quantum Field Theory. JETP Letters, 20, 194-195.

[29] Khavroshkin, O. and Tsyplakov, V. (2013) Nonlinear Seismology: The Space Component. Palmarium Academic Publishing, Saarbrücken, 516.

[30] Bakal, J. (1993) Neutrino Astrophysics. Translated from English, Mir, Moscow, 624 p.

[31] Davis Jr., R. (1996) A Review of Measurement of the Solar Neutrino Flux and Their Variation. Nuclear Physics B-Proceedings Supplements, 48, 284-298. http://dx.doi.org/10.1016/0920-5632(96)00263-0

[32] Shestopalov, I.P., Kuzhevsky, B.M. and Kharin, E.P. (2014) Correlation of Neutrino Fluxes with Seismicity of the Earth. The Hypothesis of the Possible Formation of Neutrinos on the Period of Strong Deep Earthquakes. Engineering Physics, No. 1, 5-12. 